\title{
Uso das gerontecnologias em situações de isolamento social: revisão de literatura
}

\author{
Use of gerontechnologies in social isolation situations: literature review
}

Andréa Mathes Faustino $^{1 *}$, Luiza Rosa Bezerra Callado ${ }^{1}$, Vitor Hugo Sales Ferreira ${ }^{1}$

\begin{abstract}
RESUMO
Vivenciar a solidão pode ser uma experiência psicologicamente desagradável e angustiante para quem a sente, podendo levar à exclusão social. $\mathrm{O}$ isolamento social é objetivo, porque mede o número de contatos sociais. A gerontecnologia é um campo de estudo interdisciplinar, que visa dar suporte ao envelhecimento ativo e saudável, pensando nas demandas relacionadas as perdas funcionais e aos aspectos psicológicos e sociais da velhice, no qual se desenvolvem produtos e serviços voltados para trazer soluções práticas a estas necessidades, incluindo situações de isolamento social. O presente artigo tem por objetivo identificar as gerontecnologias de apoio a idosos em situação de isolamento social, por meio de revisão de literatura nacional e internacional. Trata-se de revisão integrativa, cuja a pergunta norteadora foi: "Quais as gerontecnologias de apoio a pessoas idosas em situação de isolamento social, utilizadas no Brasil e no Mundo?" A buscas foram realizadas a partir de publicações científicas indexadas nas bases de dados: PubMed, Scopus, LILACS e Medline Scielo. O período de análise das publicações foi entre os anos de 2014 a 2019, nos idiomas inglês e português. Descritores utilizados: "idoso", "tecnologia", "isolamento social". Ao final das buscas, 33 publicações atenderam aos critérios de elegibilidade e compuseram o estudo. Diante dos achados extraídos dos estudos selecionados, percebeu-se que houve predominância de uso de tecnologias da informação para se lidar com o isolamento social entre pessoas idosas, em especial, de redes sociais ou de realidades aumentada, além das vídeo-chamadas em aplicativos. Houve uma predominância de publicações realizadas pelos Estados Unidos $(n=12)$, seguido da Austrália $(n=5)$. Apenas um artigo havia sido desenvolvido no Brasil. As gerontecnologias têm grande potencial para mitigação do isolamento social entre idosos, sendo importante incluir a participação ativa dos idosos inclusive no desenvolvimento de tais tecnologias. Apesar de promissoras e eficazes, as gerontecnologias são estratégias alternativas que não se propõem a substituir em absoluto o contato humano próximo.
\end{abstract}

Palavras-chave: Idoso; Tecnologia; Isolamento Social.

\begin{abstract}
Experiencing loneliness can be a psychologically unpleasant and distressing experience for those who feel it, which can lead to social exclusion. Social isolation is objective because it measures the number of social contacts. Gerontechnology is an interdisciplinary field of study, which aims to support active and healthy aging, thinking about the demands related to functional losses and the psychological and social aspects of old age, in which products and services are developed aimed at bringing practical solutions to these needs,
\end{abstract}

\footnotetext{
${ }^{1}$ Programa de Pós-Graduação em Desenvolvimento Sociedade e Cooperação Internacional (PPGDSCI) do Centro de Estudos Avançados Multidisciplinares (CEAM), Universidade de Brasília (UnB).

*E-mail: andreamathes@unb.br
} 
including situations of social isolation. This article aims to identify the gerontechnologies to support elderly people in a situation of social isolation, through a review of national and international literature. This is an integrative review, whose guiding question was: "What support gerontechnologies for elderly people in a situation of social isolation, used in Brazil and in the world?" Searches were performed from scientific publications indexed in databases: PubMed, Scopus, LILACS and Medline Scielo. The period of analysis of publications was from 2014 to 2019, in English and Portuguese. Descriptors used: "elderly", "technology", "social isolation". At the end of the searches, 33 publications met the eligibility criteria and composed the study. In view of the findings extracted from the selected studies, it was noticed that there was a predominance of the use of information technologies to deal with social isolation among elderly people, in particular, social networks or augmented realities, in addition to video calls in applications. There was a predominance of publications carried out by the United States ( $n=12)$, followed by Australia $(n=5)$. Only one article had been developed in Brazil. Gerontechnologies have great potential for mitigating social isolation among the elderly, and it is important to include the active participation of the elderly, including in the development of such technologies. Despite being promising and effective, gerontechnologies are alternative strategies that do not intend to replace close human contact at all.

Keywords: Aged; Technology; Social Isolation.

\section{INTRODUÇÃO}

Sentir-se só é um termo usado para um fim diferente ao de estar só, que é então denominado solitude. A ideia da solidão é um termo subjetivo. Vivenciar a solidão pode ser uma experiência psicologicamente desagradável e angustiante para quem a sente, podendo levar à exclusão social. $\mathrm{O}$ isolamento social é objetivo, porque mede o número de contatos sociais. Os sentimentos de solidão, são comuns em qualquer faixa etária, contudo podem ser mais prevalentes entre adolescentes e pessoas idosas (Azeredo; Afonso, 2016).

O movimento social intitulado isolamento social, traz a ideia de um processo de isolamento de uma pessoa e associa-se a uma série de eventos e problemas relacionados a esse movimento. Entre idosos, observam-se algumas tecnologias que podem ser associadas como estratégias eficazes capaz de favorecer um melhor prognóstico reduzindo a situação de isolamento social, como é o caso do emprego das gerontecnologias no cotidiano de pessoas idosas (Andrade, Alvim, Aparecida, 2018).

A gerontecnologia é um campo de estudo interdisciplinar, que visa dar suporte ao envelhecimento ativo e saudável, pensando nas demandas relacionadas as perdas funcionais e aos aspectos psicológicos e sociais da velhice, no qual se desenvolvem técnicas, produtos e serviços voltados para trazer soluções práticas as necessidades 
específicas desta população, como por exemplo no caso da situação de isolamento social (Andrade, Alvim, Aparecida, 2018).

Assim como o envelhecimento populacional é concernente à segunda metade do século XXI, a realidade da gerontecnologia está intimamente introjetada em todas as práticas de trabalho voltadas para a qualidade de vida de pessoas idosas, sendo um fato em vários países do mundo. A Sociedade Internacional de Gerontecnologia considera que essa área interdisciplinar contribuirá grandemente para a maximização da funcionalidade, anos de vida produtiva e independência entre idosos (Petermans; Piau, 2017).

Quanto à questão da dificuldade de adesão da população idosa ao uso de algumas tecnologias, em especial as digitais, estudos com desing participativo tem constatado que o posterior engajamento às tecnologias aumentou consideravelmente, sendo que idosos são heterogêneos, inclusive na resistência ou não, e na assimilação de novas tecnologias. Considera-se também que, conforme as gerações forem envelhecendo, a geração que já nasceu nativa digital também terá sua inserção de forma mais intuitiva (Petermans; Piau, 2017; Merkel; Kurshaski, 2018).

Neste sentido, o presente artigo tem por objetivo identificar as gerontecnologias de apoio à idosos em situação de isolamento social, por meio da revisão de literatura nacional e internacional.

\section{MÉTODOS}

Trata-se de revisão integrativa de literatura, a qual permite por meio do seu método ampliar o escopo de estudos analisados, pois podem ser incluídos tanto estudos experimentais e não-experimentais, além de dados da literatura teórica e empírica (Souza; Silva; Carvalho; 2010).

A pergunta norteadora foi "Quais as gerontecnologias de apoio a pessoas idosas em situação de isolamento social utilizadas no Brasil e no Mundo? ”. Foram utilizados os seguintes critérios de inclusão: artigos que trouxessem estudos com participantes acima dos 60 anos, somente artigos com textos completos disponíveis on-line, nos idiomas inglês e português, publicados no período entre os anos de 2014 a 2019. A obtenção dos artigos selecionados ocorreu por meio de acesso a periódicos disponíveis em meio eletrônico durante os meses de junho a agosto de 2020. 
Foram utilizadas as bases de dados PubMed, Scopus, LILACS e Medline Scielo. Os descritores controlados utilizados na pesquisa foram retirados da Medical Subject Headings (MESH) da National Library of Medicine (NLM) dos Estados Unidos e dos Descritores em Saúde (DECS) da Biblioteca Virtual em Saúde (BVS) do Brasil, sendo eles: "idoso", "tecnologia", "isolamento social", "aged", "technology”, "social isolation”.

Figura 1. Diagrama de seleção dos estudos que fizeram parte da revisão de literatura.

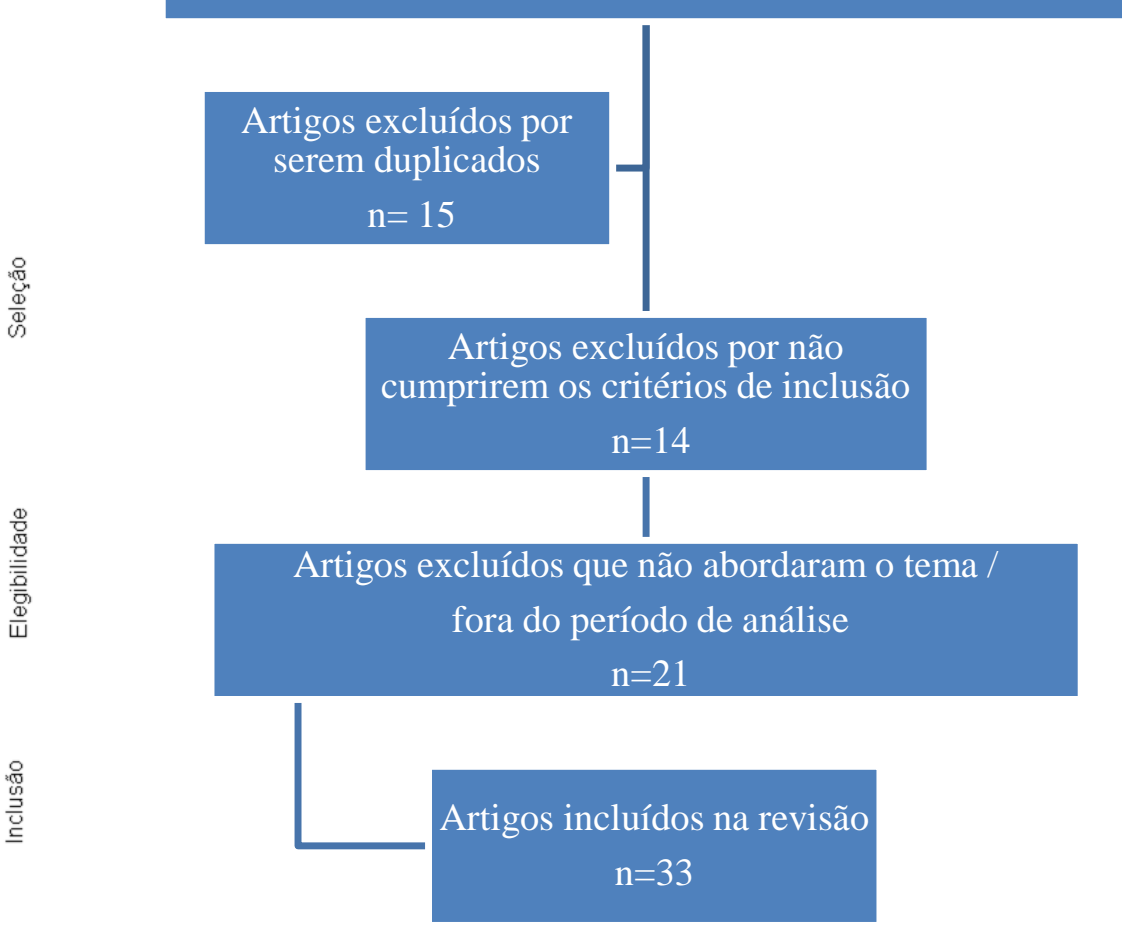

Fonte: Faustino, Callado, Ferreira (2020).

Com os resultados obtidos, foi feita uma divisão em três etapas organizacionais: a $1^{\text {a }}$ etapa foi relacionada a busca de artigos nas bases de dados selecionadas com um resultado de 83 artigos ao total. Na $2^{\text {a }}$ etapa foi utilizado da exclusão de artigos duplicados 
restando um total de 68 artigos. Por último, na $3^{\text {a }}$ etapa foi feita a leitura dos artigos e realizada a exclusão daqueles que não se adequam aos critérios de inclusão, que não se referiam à população-alvo do estudo que totalizaram 14 artigos. Também foram excluídos os artigos que não se referiam ao isolamento social entre idosos $(n=19)$ e aqueles que não eram pertencentes ao período de 2014 a 2019 (n=2). Ao final a amostra de artigos incluídos totalizou 33 artigos (Figura 1).

\section{RESULTADOS}

No Quadro 1 estão apresentados os dados referentes a identificação dos autores, ano, local, desenho e tipo de amostra utilizada nos artigos incluídos.

Quanto ao tipo de estudo 30,3\% ( $\mathrm{n}=10)$ eram Estudos clínicos, 15,15\% (n=5) Estudo de caso e 12,12\% (n=4) Revisão de Literatura, entre outros tipos. Em relação a origem 36,36\% ( $\mathrm{n}=12)$ são dos Estados Unidos da América (EUA), 15,15\% ( $\mathrm{n}=5)$ provenientes da Austrália e 12,12\% (n=4) da China. Apenas um dos estudos encontrados era de origem brasileira. $\mathrm{O}$ ano com maior número de publicações sobre o tema foi em 2017 com 30,3\% ( $n=10)$ dos artigos analisados seguido do ano de 2016 com 27,3\% (n=9). A respeito do tipo de amostra a maioria eram exclusivamente com idosos, ou seja, pessoas acima dos 60 anos, vivendo em ambiente da comunidade, ou em muitos casos residindo sozinhos. Também foi observado como participantes dos estudos cuidadores de idosos, profissionais da saúde além de artigos científicos e até um robô (Quadro 1).

Quadro 1. Distribuição dos artigos segundo autor, ano, local, desenho e tipo de $\operatorname{amostra}(n=33)$.

\begin{tabular}{|c|c|c|c|c|c|}
\hline $\mathbf{N}^{\circ}$ & Autor & Ano & Local & Desenho & Tipo de Amostra \\
\hline 1. & PIAU et al & 2014 & $\begin{array}{c}\text { Toulouse, } \\
\text { França }\end{array}$ & Revisão de literatura & 1.246 artigos científicos \\
\hline 2. & BOMAN et al & 2014 & $\begin{array}{c}\text { Estocolmo, } \\
\text { Suécia }\end{array}$ & Estudo observacional & 4 idosos com demência \\
\hline 3. & TSAI et al & 2015 & Boston, EUA & $\begin{array}{c}\text { Estudo prospectivo de } \\
\text { coorte }\end{array}$ & $\begin{array}{c}72.607 \text { idosas (mulheres } \\
\text { enfermeiras) }\end{array}$ \\
\hline
\end{tabular}




\begin{tabular}{|c|c|c|c|c|c|}
\hline 4. & WAYCOTT et al & 2015 & $\begin{array}{l}\text { Victoria, } \\
\text { Australia }\end{array}$ & $\begin{array}{l}\text { Ensaio clínico não- } \\
\text { randomizado }\end{array}$ & $\begin{array}{l}\text { Idosos atendidos em home care } \\
\text { com autocuidado insuficiente }\end{array}$ \\
\hline 5. & $\begin{array}{c}\text { GUSTAFSON et } \\
\text { al }\end{array}$ & 2015 & Madison, EUA & $\begin{array}{l}\text { Ensaio clínico } \\
\text { randomizado } \\
\text { prospectivo }\end{array}$ & 450 idosos (residem sozinhos) \\
\hline 6. & CHESLER et al & 2015 & $\begin{array}{l}\text { Ballarat, } \\
\text { Australia }\end{array}$ & $\begin{array}{c}\text { Estudo } \\
\text { qualiquantitativo, quasi- } \\
\text { experimental }\end{array}$ & $\begin{array}{c}158 \text { idosos (com depressão e } \\
\text { independentes) }\end{array}$ \\
\hline 7. & HICKEN et al & 2015 & Utah, EUA & Estudo de caso controle & $\begin{array}{c}231 \text { cuidadores de idosos rurais } \\
\text { (veteranos de guerra e com } \\
\text { demência) }\end{array}$ \\
\hline 8. & $\begin{array}{l}\text { KOCESKI, } \\
\text { KOCESKA }\end{array}$ & 2016 & $\begin{array}{l}\text { Macedônia, } \\
\text { Grécia }\end{array}$ & $\begin{array}{l}\text { Ensaio clínico não- } \\
\text { randomizado }\end{array}$ & $\begin{array}{l}30 \text { idosos com vulnerabilidade } \\
\text { e } 5 \text { cuidadores profissionais }\end{array}$ \\
\hline 9. & ZHONG et al & 2016 & $\begin{array}{c}\text { Rochester, Nova } \\
\text { York, EUA }\end{array}$ & $\begin{array}{c}\text { Análise de dados } \\
\text { secundária }\end{array}$ & 14.999 idosos \\
\hline 10. & $\begin{array}{c}\text { DOLOVITCH et } \\
\text { al }\end{array}$ & 2016 & $\begin{array}{l}\text { Hamilton, } \\
\text { Canadá }\end{array}$ & $\begin{array}{l}\text { Ensaio clínico } \\
\text { randomizado }\end{array}$ & 316 idosos (70 anos ou mais) \\
\hline 11. & LOI et al & 2016 & $\begin{array}{l}\text { Victoria, } \\
\text { Australia }\end{array}$ & $\begin{array}{l}\text { Ensaio clínico não- } \\
\text { randomizado }\end{array}$ & $\begin{array}{c}5 \text { idosos (residentes em hospital } \\
\text { psiquiátrico) }\end{array}$ \\
\hline 12 . & MEZUK et al & 2016 & $\begin{array}{c}\text { Los Angeles, } \\
\text { EUA }\end{array}$ & Estudo de caso controle & 441 idosos (residem sozinhos) \\
\hline 13. & PETERSEN et al & 2016 & $\begin{array}{c}\text { Portland (EUA), } \\
\text { San Diego } \\
\text { (EUA), } \\
\text { Nashville (EUA) } \\
\text { e Perth } \\
\text { (Australia) }\end{array}$ & $\begin{array}{l}\text { Estudo de coorte } \\
\text { transversal }\end{array}$ & $\begin{array}{c}26 \text { idosos (com mais de } 70 \text { anos } \\
\text { independentes) }\end{array}$ \\
\hline 14. & CHOPIK & 2016 & Michigan, EUA & $\begin{array}{c}\text { Análise de dados } \\
\text { secundária }\end{array}$ & $\begin{array}{l}591 \text { idosos solitários inscritos } \\
\text { em questionário nacional de } \\
\text { saúde }\end{array}$ \\
\hline 15 . & CHEN, SCHULZ & 2016 & $\begin{array}{l}\text { Hong Kong, } \\
\text { China }\end{array}$ & $\begin{array}{c}\text { Revisão sistemática de } \\
\text { literatura }\end{array}$ & 25 artigos científicos \\
\hline 16. & SACKER et al & 2016 & $\begin{array}{l}\text { Londres, } \\
\text { Inglaterra }\end{array}$ & $\begin{array}{l}\text { Análise de dados } \\
\text { secundária }\end{array}$ & $\begin{array}{l}\text { Dados do UK Household } \\
\text { Longitudinal Study }\end{array}$ \\
\hline 17. & MYHRE et al & 2017 & Arizona, EUA & $\begin{array}{l}\text { Ensaio clínico } \\
\text { randomizado }\end{array}$ & 48 idosos \\
\hline
\end{tabular}




\begin{tabular}{|c|c|c|c|c|c|}
\hline 18. & ZHONG et al & 2017 & $\begin{array}{l}\text { Hangzhou, } \\
\text { China }\end{array}$ & $\begin{array}{l}\text { Estudo prospectivo de } \\
\text { coorte }\end{array}$ & 2995 idosos \\
\hline 19. & BERG et al & 2017 & $\begin{array}{l}\text { Victoria, } \\
\text { Australia }\end{array}$ & Estudo de caso controle & $\begin{array}{c}60 \text { idosos (residentes em zonas } \\
\text { rurais) }\end{array}$ \\
\hline 20. & ANTUNES et al & 2017 & $\begin{array}{l}\text { São Paulo, } \\
\text { Brasil }\end{array}$ & $\begin{array}{l}\text { Estudo prospectivo } \\
\text { randomizado }\end{array}$ & 23 idosos \\
\hline 21. & DAMANT et al & 2017 & Michigan, EUA & Revisão de literatura & 91 artigos científicos \\
\hline 22. & TOMASINO et al & 2017 & Chicago, EUA & $\begin{array}{l}\text { Ensaio clínico não- } \\
\text { randomizado }\end{array}$ & 47 idosos com depressão \\
\hline 23. & CLAIR et al & 2017 & $\begin{array}{c}\text { Awckland, Nova } \\
\text { Zelândia }\end{array}$ & $\begin{array}{c}\text { Revisão sistemática de } \\
\text { literatura }\end{array}$ & 9 artigos científicos \\
\hline 24. & CHI et al & 2017 & $\begin{array}{l}\text { Washington, } \\
\text { EUA }\end{array}$ & $\begin{array}{l}\text { Análise de dados } \\
\text { secundária }\end{array}$ & $\begin{array}{l}10 \text { mulheres idosas (entre } 68 \mathrm{e} \\
89 \text { anos independentes) }\end{array}$ \\
\hline 25. & $\begin{array}{c}\text { CRÉTEL- } \\
\text { DURAND et al }\end{array}$ & 2017 & $\begin{array}{l}\text { Marseille, } \\
\text { França }\end{array}$ & $\begin{array}{l}\text { Ensaio clínico } \\
\text { randomizado }\end{array}$ & $\begin{array}{l}160 \text { Idosos com mais de } 70 \\
\text { anos, independentes e com } \\
\text { câncer em estágio avançado }\end{array}$ \\
\hline 26. & HAO et al & 2017 & África do Sul & $\begin{array}{c}\text { Análise de dados } \\
\text { secundária }\end{array}$ & $\begin{array}{l}422 \text { idosos (sul africanos } \\
\text { isolados, com depressão) }\end{array}$ \\
\hline 27. & CZAJAH et al & 2018 & Florida, EUA & $\begin{array}{l}\text { Ensaio clínico } \\
\text { randomizado }\end{array}$ & $\begin{array}{l}150 \text { idosos (residem sozinhos e } \\
\text { com comprometimento visual) }\end{array}$ \\
\hline 28. & NEVILLE et al & 2018 & $\begin{array}{c}\text { Awckland, Nova } \\
\text { Zelândia }\end{array}$ & $\begin{array}{l}\text { Estudo de coorte } \\
\text { transversal }\end{array}$ & $\begin{array}{c}614 \text { homens idosos (60 e } 79 \\
\text { anos) }\end{array}$ \\
\hline 29. & SIN et al & 2018 & $\begin{array}{l}\text { Hong Kong, } \\
\text { China }\end{array}$ & Estudo de caso controle & 25 idosos \\
\hline 30. & JING et al & 2018 & Tangshan, China & $\begin{array}{l}\text { Ensaio clínico } \\
\text { randomizado }\end{array}$ & 120 idosos \\
\hline 31. & ZAMIR et al & 2018 & $\begin{array}{c}\text { Devon, } \\
\text { Inglaterra }\end{array}$ & $\begin{array}{c}\text { Pesquisa ativa } \\
\text { colaborativa } \\
\text { (collaborative action } \\
\text { research) }\end{array}$ & $\begin{array}{c}11 \text { funcionários do serviço } \\
\text { nacional de saúde (NHS), } 21 \\
\text { staffs de casas de cuidados e } 34 \\
\text { idosos ( } 19 \text { residentes em casas } \\
\text { de cuidados, } 15 \text { admitidos em } \\
\text { hospital vindos de casa de } \\
\text { cuidados) }\end{array}$ \\
\hline 32. & KOCESKA et al & 2019 & $\begin{array}{l}\text { Macedônia, } \\
\text { Grécia }\end{array}$ & Estudo de caso controle & $\begin{array}{c}26 \text { idosos e } 5 \text { cuidadores } \\
\text { profissionais }\end{array}$ \\
\hline 33. & ARLATI et al & 2019 & Milão, Itália & Relato de experiência & Robô \\
\hline
\end{tabular}


Fonte: Faustino, Callado, Ferreira (2020).

O assunto do isolamento social entre a população idosa tem sido estudado há pouco tempo, em associação com o envelhecimento da população mundial. Se a discussão em si é atual, as gerontecnologias desenvolvidas para o enfrentamento do isolamento social são ainda mais recentes.

Ao analisar os 33 artigos incluídos, constata-se a predominância do uso de tecnologias da informação para lidar com o isolamento social entre pessoas idosas, em especial, de redes sociais ou de realidades aumentada. Somente por meio dessa revisão, não foi possível identificar o porquê dessa escolha preferencial por tecnologias duras da área de comunicação e informação.

\section{DISCUSSÃO}

Todo campo de estudo a respeito do isolamento social entre idosos tem sido explorado há apenas alguns anos atrás. As estratégias para minimizar esse fenômeno, inclusive, são ainda mais recentes. Para Zhong et al (2015), em sua análise de dados secundária de 14.999 chineses idosos, a solidão e o isolamento social são experienciadas de forma diferente entre homens e mulheres, entre indivíduos com declínio cognitivo ou sem ele, sendo para os primeiros o de maior risco. O estudo de coorte transversal de Neville et al (2018), com 614 homens idosos neozelandeses concorda e afirma que para os homens, o risco de solidão é ainda maior, fato também apontado por Wright-St Clair et al, 2017. Ser solteiro é fator de risco para solidão e isolamento social tanto para homens quanto para mulheres, segundo Mezuk et al (2016), tendo os homens risco ainda substancialmente superior.

As gerontecnologias são vistas como alternativas benéficas pela maioria dos estudos encontrados por propiciar melhor conexão com o mundo exterior, ganho de apoio social, engajamento de atividades de interesse e ganho de confiança. Há expressivas constatações de que as gerontecnologias foram bem recebidas e bem incorporadas pelos idosos nas amostras incluidas nas pesquisas analisadas (Dolovitch et al, 2016; Arlati et al, 2019; Koceska et al, 2018; Piau et al, 2014; Koceski e Koceska, 2015; Boman et al, 2014; Tomasino et al, 2017; Sacker et al, 2017; Chi et al, 2017; Chopik, 2016; Chen e 
Schulz, 2016; Gustafson et al, 2015; Hicken et al, 2016; Crétel-Durand et al, 2017; Chi et al., 2017; Petersen et al, 2017; Czaja et al, 2016; Sacker et al, 2016).

Muitas das gerontecnologias mais empregadas (videochamadas e outros meios de comunicação, redes sociais virtuais, games, dispositivos afetivos/ ortopédicos e/ou robôs companheiros) se mostraram efetivas na redução do isolamento social dos idosos (Berg et al, 2017; Piau et al, 2014; Damant et al, 2017; Sacker et al, 2017; Chi et al, 2017; Creteh-durand et al, 2017; Chen e Schulz, 2016; Zamir et al, 2018; Chesler et al, 2015; Chopik, 2016; Hicken et al, 2016; Crétel-Durand et al, 2017; Chi et al., 2017; Petersen et al, 2017; Czaja et al, 2016; Sacker et al, 2016; Koceski e Koceska, 2016; Berg et al, 2017; Arlati et al, 2019). Esses achados são de grande importância, haja vista a significativa relação direta entre isolamento social de idosos e suicídio relatada por Tsai et al (2015) e Hao et al (2017).

As videochamadas se mostraram especialmente incorporáveis no cotidiano dos idosos, segundo Damant et al (2017), Boman et al (2014), Zamir et al (2018) e Petersen et al, 2017. Alguns idosos relataram terem considerado "útil" e "agradável", a utilização das videochamadas para manutenção de diálogos com familiares e amigos, apesar de algumas dificuldades iniciais (Damant et al, 2017; Boman et al, 2014; Petersen et al, 2017; Berg, et al 2017). O trabalho de Boman et al (2014), que avaliou engajamento de idosos demenciados às videochamadas, também apresentou resultados positivos.

Há poucas evidências científicas quanto à eficácia das gerontecnologias para diminuir o isolamento social de pessoas idosas entre aqueles que são mais fragilizados ou em situação de vulnerabilidades. Dos 33 estudos analisados, quatro tratavam de alguns desses grupos de idosos mais vulneráveis. Antunes et al (2017) em seu trabalho sobre games de realidade virtual entre idosos com limitações de locomoção, observou incrementos na adaptação a ambientes diversos, sem perda de segurança do usuário para risco de quedas.

$\mathrm{Na}$ avaliação de idosos em situação de vulnerabilidade, usuários de serviços de home care, observados no estudo de Waycott et al (2015), a preocupação foi com a continuidade do suporte a serviços de comunicação voltados a idosos e seus familiares, demonstrando um marcador de exclusão socioeconômico no uso deste tipo de gerontecnologias, sendo a mesma preocupação manifestada por Chi et al (2017) e Sacker et al (2016) em suas pesquisas. 
A revisão de literatura de Piau et al (2014) sobre uso de dispositivos cujo objetivo era amenizar a solidão de idosos, especialmente idosos fragilizados, também apontou diversas limitações nos trabalhos realizados até então. Todos os quatro estudos concordam quanto à necessidade de mais trabalhos que incluam idosos fragilizados e/ou em situação de vulnerabilidade em suas amostras (Piau et al, 2014; Waycott et al, 2015; Antunes et al, 2017; Boman et al, 2014).

Entre idosos com transtornos de ansiedade e de depressão residente nos EUA e na Nova Zelândia, as redes de relações sociais virtuais, especialmente Facebook, influenciaram negativamente, piorando quadros de transtornos afetivos, sendo então evitadas por eles. A tecnologia vista por esse mesmo grupo como mais benéfica foram as videochamadas (Myhre et al, 2016; Neville et al, 2018; Tomasino et al, 2017; Berg et al, 2017). Myre et al (2016), Chen e Shulz (2016), Sin et al (2018) e Jin et al (2018) afirmaram ainda que redes sociais virtuais em um curto prazo contribuíram para o desenvolvimento de habilidades cognitivas complexas entre idosos, mas não se mostraram eficientes em reduzir o isolamento social.

Para Loi et al (2016) e Zamir et al (2018), algumas das possíveis barreiras para implementação e engajamento de gerontecnologias com finalidade de reduzir efetivamente o isolamento social de idosos estão: alta rotatividade de equipe profissional e atitude de negação ao uso de tais tecnologias, aversão por risco de aprender novas habilidades por parte dos idosos, design e usabilidades das tecnologias inadequados para a população idosa e falta de adesão também por parte dos familiares.

\section{CONSIDERAÇÕES FINAIS}

As gerontecnologias têm grande potencial para mitigação do isolamento social entre idosos, quando adequadas às necessidades específicas, sendo importante incluir a participação ativa dos idosos inclusive no desenvolvimento de tais tecnologias. Cenários ideais pressuporiam a presença real e significativa durante a maior frequência possível na vida dessas pessoas idosas.

As diversas demandas as quais filhos, amigos e demais familiares têm que cumprir e que tomam tempo de suas agendas e rotinas potencialmente comprometem esse vínculo. Apesar de promissoras e eficazes, as gerontecnologias são estratégias alternativas que não 
se propõem a substituir em absoluto o contato humano próximo e constante, sempre que possível.

\section{REFERÊNCIAS}

ANDRADE P. O. P. C.; ALVIM, T.; APARECIDA, N. Jogo de tabuleiro: uma gerontecnologia na clínica do cuidado de enfermagem. Revista Brasileira de Enfermagem, v. 71, suppl 2, p. 818-826, 2018.

ANTUNES, T.; OLIVEIRA, A.; CROCETTA, T. B.; et al. Computer classes and games in virtual reality environment to reduce loneliness among students of an elderly reference center: Study protocol for a randomised cross-over design. Medicine, v.96, n.10, e5954, 2017.

ARLATI, S.; COLOMBO, V.; SPOLADORE, D.; et al. A Social Virtual Reality-Based Application for the Physical and Cognitive Training of the Elderly at Home. Sensors, v.19, n.2, p. 261, 2019.

AZEREDO, Z. de A. S.; AFONSO, M. A. N. Loneliness from the perspective of the elderly. Revista Brasileira de Geriatria e Gerontologia, v. 19, n. 2, p. 313-324, 2016.

BERG, T.; WINTERTON, R.; PETERSEN, M.; et al. 'Although we're isolated, we're not really isolated': The value of information and communication technology for older people in rural Australia. Australas J Ageing, v. 36, n.4, 313-317, 2017.

BOMAN, I.L.; LUNDBERG, S.; STARKHAMMAR, S. et al. Exploring the usability of a videophone mock-up for persons with dementia and their significant others. BMC Geriatrics, v. 14, n. 49, p.1-11, 2014.

CHEN, Y. R.; SCHULZ, P. J. The Effect of Information Communication Technology Interventions on Reducing Social Isolation in the Elderly: A Systematic Review.

Journal of Medical Internet Research, v. 18, n. 18, p.1-11, 2016.

CHESLER, J.; MCLAREN, S.; KLEIN, B.; et al.The effects of playing Nintendo Wii on depression, sense of belonging and social support in Australian aged care residents: a protocol study of a mixed methods intervention trial. BMC Geriatrics, v. 15, p. 106$114,2015$.

CHI, N.C.; SPARKS, O.; LIN, S.Y.; et al. Pilot testing a digital pet avatar for older adults. Geriatric Nursing, v. 36, n. 6, p. 542-547, 2017.

CHOPIK, W.J. The Benefits of Social Technology Use Among Older Adults Are Mediated by Reduced Loneliness. Cyberpsychol Behav Soc Netw, v.19, n.9, p.551-6, 2016. 
CRÉTEL-DURAND, E.; NOUGUERÈDE, E.; LE CAER, H., et al. PREDOMOS study, impact of a social intervention program for socially isolated elderly cancer patients: study protocol for a randomized controlled trial. Trials, v. 18., n.1, p. 174-185, 2017.

CZAJA, S.J.; BOOT, W.R.; CHARNESS, N.; et al. Improving Social Support for Older Adults Through Technology: Findings From the PRISM Randomized Controlled Trial. Gerontologist, v. 58, n. 3, p.467-477, 2018.

DAMANT, J.; KNAPP, M.; FREDDOLINO, P.; et al. Effects of digital engagement on the quality of life of older people. Health and Social Care in the Community, v.25, n.6, p.1679-1703, 2017.

DOLOVICH, L.; OLIVER, D.; LAMARCHE, L.; et al. A protocol for a pragmatic randomized controlled trial using the Health Teams Advancing Patient Experience: Strengthening Quality (Health TAPESTRY) platform approach to promote personfocused primary healthcare for older adults. Implementation Science, v.11, p.49, 2016.

GUSTAFSON, D.H.; MCTAVISH, F.; GUSTAFSON, D.H.; et al. The effect of an information and communication technology (ICT) on older adults' quality of life: study protocol for a randomized control trial. Trials, v.16, p. 191, 2015.

HAO, G.; BISHWAJIT, G.; TANG, S.; et al. Social participation and perceived depression among elderly population in South Africa. Clinical Interventions in Aging, v. 17, n. 12, p. 971-976, 2017.

HICKEN BL, DANIEL C, LUPTAK M.; et al. Supporting Caregivers of Rural Veterans Electronically (SCORE). The Journal of Rural Health, v. 33, n.3, p. 305-313, 2015.

JING, L.; JIN, Y.; ZHANG X.; et al. The effect of Baduanjin qigong combined with CBT on physical fitness and psychological health of elderly housebound. Medicine, $v$. 97, n. 51, p.13654-61, 2018.

KOCESKA, N.; KOCESKI, S.; BEOMONTE ZOBEL, P.; et al. A Telemedicine Robot System for Assisted and Independent Living. Sensors, v.19, n.4, 834, 2019.

KOCESKI, S.; KOCESKA, N. Evaluation of an Assistive Telepresence Robot for Elderly Healthcare. J Med Syst. V.40, n.5, p.121, 2016.

LOI, S.M.; HODSON, S.; HUPPERT, D.; et al. Can a short internet training program improve social isolation and self-esteem in older adults with psychiatric conditions? International Psychogeriatrics, v.28, n.10, p.1737-1740, 2016.

MERKEL, S.; KURCHASKI, A. Participatory Design in Gerontotechnology: a Systematic Literature Review. The Gerontologist, Oxford, v. 9, n. 1, p. e16-e25, 2018.

MEZUK,B.; CHOI, M.; DESANTIS, A.S.; et al. Loneliness, Depression, and Inflammation: Evidence from the Multi-Ethnic Study of Atherosclerosis. PLoS One, v.11, n.7, p.e0158056, 2016. 
MYHRE, J.W.; MEHL, M.R.; GLISKY, E.L. Cognitive Benefits of Online Social Networking for Healthy Older Adults. J Gerontol B Psychol Sci Soc Sci, v. 72, n.5, p.752-760, 2017.

NEVILLE, S.; ADAMS, J.; MONTAYRE, J.; et al. Loneliness in Men 60 Years and Over: The Association With Purpose in Life. American Journal of Men's Health, v.12, n.4, p.730-739, 2018.

PETERMANS, J.; PIAU, A. Gerontotechnology: Don't miss the train, but which is the right carriage? Editorial Journal Europeian Geriatric Medicine, Tolousse, v. 8, p. 281-283, 2017.

PETERSEN, J.; THIELKE, S.; AUSTIN, D.; et al. Phone behaviour and its relationship to loneliness in older adults. Aging Ment Health, v. 20, n. 10., p. 1084-1091, 2016.

PIAU, A.; CAMPO, E.; RUMEAU, P.; et al. Aging society and gerontechnology: a solution for an independent living? J Nutr Health Aging, v.18, n.1, p.97-112, 2014.

SACKER, A.; ROSS, A.; MACLEOD, C.A.; et al. Health and social exclusion in older age: evidence from Understanding Society, the UK household longitudinal study.

Epidemiol Community Health, v. 71, n. 7, p. 681-690, 2016.

SIN, E.L.L.; LIU, H.L.; LEE, S.H.; et al. The relationships between brain structural changes and perceived loneliness in older adults suffering from late-life depression. Int J Geriatr Psychiatry, v. 33, p. 606-612, 2018.

SOUSA, M. T.; SILVA, M. D.; CARVALHO, R. Revisão integrativa: o que é e como fazer. Einstein, v.8, n.1, p. 102-106, 2010.

TOMASINO, K.N.; LATTIE, E.G.; HO, J.; et al. Harnessing Peer Support in an Online Intervention for Older Adults with Depression. American Journal of Geriatric and Psychiatry, v. 25, n. 10, p. 1109-1119, 2017.

TSAI, A.C.; LUCAS, M.; KAWACHI, I. Association between social integration and suicide among women in the United States. JAMA Psychiatry, v.72, n.10, p.987-993, 2015.

WAYCOTT, J.; MORGANS, A.; PEDELL, S.; et al. Ethics in Evaluating a Sociotechnical Intervention With Socially Isolated Older Adults. Qualitative Health Research, v. 25, n. 11, p.1518-1528, 2015.

WRIGHT-ST CLAIR, V.A.; NEVILLE, S.; FORSYTH, V.; et al. Indigenous Ageing Research Feature Integrative review of older adult loneliness and social isolation in Aotearoa/New Zealand. Australasian Journal on Ageing, v. 36, n. 2, p.114-123, 2017.

ZAMIR, S.; HENNESSY, C.H.; TAYLOR, A.H.; et al. Video-calls to reduce loneliness and social isolation within care environments for older people: an implementation study using collaborative action research. BMC Geriatrics, v. 18, p.62-75, 2018. 
ZHONG, B.L.; CHEN, S.L.; CONWELL, Y. Effects of transient versus chronic loneliness on cognitive function in older adults: Findings from the Chinese Longitudinal Healthy Longevity Survey. Am J Geriatr Psychiatry, v.24, n.5, p.389-398, 2016.

ZHONG, B.L.; CHEN, S.L.; TU, X.; et al. Loneliness and Cognitive Function in Older Adults: Findings From the Chinese Longitudinal Healthy Longevity Survey. J Gerontol B Psychol Sci Soc Sci, v. 72, n. 1, p. 120-128, 2017. 